\title{
$\eta$ Carinae - The outer ejecta
}

\author{
K. Weis \\ Astronomisches Institut der Ruhr-Universität Bochum, Universitätsstr. 150, 44780 Bochum, Germany
}

\begin{abstract}
$\eta$ Carinae is a unique object among the most massive evolved stars in the LBV phase. The central object(s) is(are) surrounded by a complex circumstellar nebula ejected during more than one eruption in the 19th century. Beyond the well-defined edges of its famous bipolar nebula, the Homunculus, are additional nebulous features referred to as the outer ejecta. The outer ejecta contains a large variety of structures of very different sizes and morphologies distributed in a region $0.67 \mathrm{pc}$ in diameter. Individual features in the outer ejecta are moving extremely fast, up to $3200 \mathrm{~km} / \mathrm{s}$, in general the expansion velocities are between $400-900 \mathrm{~km} / \mathrm{s}$. A consequence of these high velocities is that structures in the outer ejecta interact with the surrounding medium and with each other. The strong shocks that arise from these interactions give rise to soft $\mathrm{X}$-ray emission. The global expansion pattern of the outer ejecta reveals an overall bipolar distribution, giving a symmetric structure to its morphologically more irregular appearance. The long, highly collimated filaments, called strings, are particularly unusual. The material in the strings follows a Hubble-flow and appears to originate at the central object.
\end{abstract}

Individual Objects: $\eta$ Car, AG Car, HR Car, P Cyg

\section{The outer ejecta - morphology, kinematics and X-ray emission}

Historic background

Van den Bos (1938) noticed for the first time extended nebular emission around $\eta$ Car, Gaviola (1950) published the first images and due to its human-like appearance called the nebula the Homunculus. He also identified some bright regions around the Homunculus. Thackeray (1949) sketched the nebula and speculated also about a fainter outer nebula or shell. In 1976 Walborn identified structures outside the Homunculus, he called them N, E, S and W condensations, $\mathrm{S}$ ridge and $\mathrm{W}$ arc.

The morphology of the nebula

Today HST images show that $\eta$ Car is surrounded by a bipolar inner nebular, the Homunculus, which consists of two lobes and an equatorial disk, its longest axis is $19^{\prime \prime}(0.2 \mathrm{pc})$. Further out a larger clumpy irregularly shaped outer nebulae with a diameter of at least $60^{\prime \prime}(0.67 \mathrm{pc})$ is visible and called the outer ejecta. Individual structures in the outer ejecta can be described as filaments, jets, arcs, bullets or knots and strings. The electron density in the outer ejecta is about $10^{4} \mathrm{~cm}^{-3}$, assuming a typical electron temperature of $1400 \mathrm{~K}$ and a volume filling factor for the clumps, a total mass of $2-4 \mathrm{M}_{\odot}$ can be derived for the outer ejecta. 

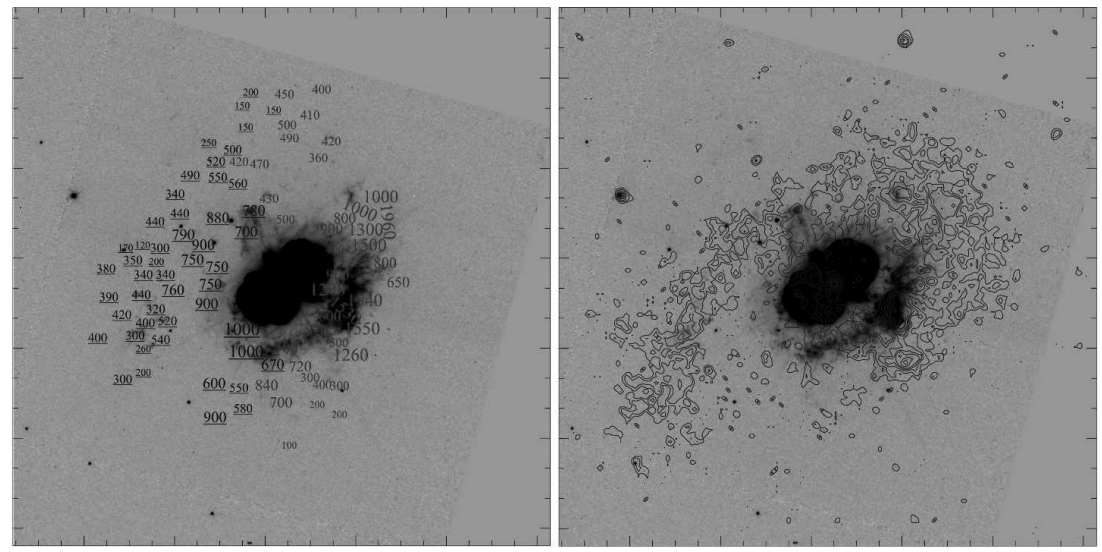

Figure 1: In grey scale an HST image of the nebula around $\eta$ Car is shown. Left: Kinematic analysis shows the bipolar expansion pattern (blueshifted values underlined). Right: In contours the X-ray emission.

\section{Kinematic analysis}

Early measurements (e.g. Walborn 1976) show that expansion velocities in the outer ejecta reach up to more than $1000 \mathrm{~km} / \mathrm{s}$. Weis et al.'s (2001) systematic study revealed unexpected results for the kinematic behaviour of the individual structures with expansion velocities ranging from roughly $-1000 \mathrm{~km} / \mathrm{s}$ to $3200 \mathrm{~km} / \mathrm{s}$, with the majority between $400-900 \mathrm{~km} / \mathrm{s}$ (see Fig. 1). While the observed features in the outer ejecta show a more or less irregular and random distribution, the red and blueshifted velocities (Fig. 1, left) show a systematic bipolar expansion pattern. Structures in the (south-)east are blueshifted, those in the (north-)west redshifted. The outer ejecta follows the same symmetry and has the same axis as the Homunculus (southern lobe tilted towards the observer, northern away).

\section{The strings}

Most amazing in the outer ejecta are long, highly collimated linear features identified by visual inspection of HST images and now labeled string 1, 2, 3, 4, 5 (Weis et al. 1999). The length of the strings varies from the shortest $0.044 \mathrm{pc}$ (string 5) to $0.177 \mathrm{pc}$ for the longest (string 1). The width is 0.002 to $0.003 \mathrm{pc}$, yielding a length-to-width ratio of 31 to 70. Kinematic measurements show that all strings show an increasing radial velocity towards their outer end, a perfect Hubble-flow.

\section{X-ray emission}

An overlay of an HST with a CHANDRA image (soft X-ray emission 0.6-1.2 keV) shows a very good spatial agreement between the optical and diffuse X-ray emission (Fig. 1, right). The X-ray emission exhibits a hook shape structure, composed of smaller substructures. Across the hook, a bridge projects onto the Homunculus from the south to the north-east. The X-ray emission of the outer ejecta is created by shocks due to collisions of the individual structures with the ISM and with each other. Determined from postshock temperatures, the postshock velocities necessary to reproduce the X-ray emission are roughly $670-760 \mathrm{~km} / \mathrm{s}$, exactly what is observed. It can be concluded that the X-ray image of the outer ejecta displays the shock fronts of the interacting material. 


\section{Other LBV nebula, some facsimiles of the outer ejecta}

$\eta$ Car is not the only LBV surrounded by a nebula. A statistic of known, and resolved, LBV nebulae shows that $50 \%$ of the nebulae are bipolar to some degree, about $40 \%$ are roughly spherical, and less than 10\% appear completely irregular. Size and kinematics show that the nebula around HR Car appears as an older twin of the Homunculus (Weis et al. 1997). HR Car's nebula but also the nebulae around AG Car and P Cygni show larger components which could be regarded as facsimiles of the outer ejecta.

A complete review article on the outer ejecta (Weis 2009) will appear in " $\eta$ Carinae and the supernovae imposters", eds. K. Davidson and R. M. Humphreys, Springer-Verlag.

Acknowledgments. I thank D.J. Bomans and W.J. Duschl for countless discussions and their input.

\section{References}

van den Bos, W. H. 1938, Union Obs. Circ., 100, 522

Gaviola, E. 1950, ApJ, 111, 408

Thackeray, A. D. 1949, The Observatory, 69, 31

Walborn, N. R. 1976, ApJ, 204, L17

Weis, K., Duschl, W. J., Bomans, D. J., et al. 1997, A\&A, 320, 568

Weis, K., Duschl, W. J., \& Chu, Y.-H. 1999, A\&A, 349, 467

Weis, K., Duschl, W. J., \& Bomans, D. J. 2001, A\&A, 367, 566

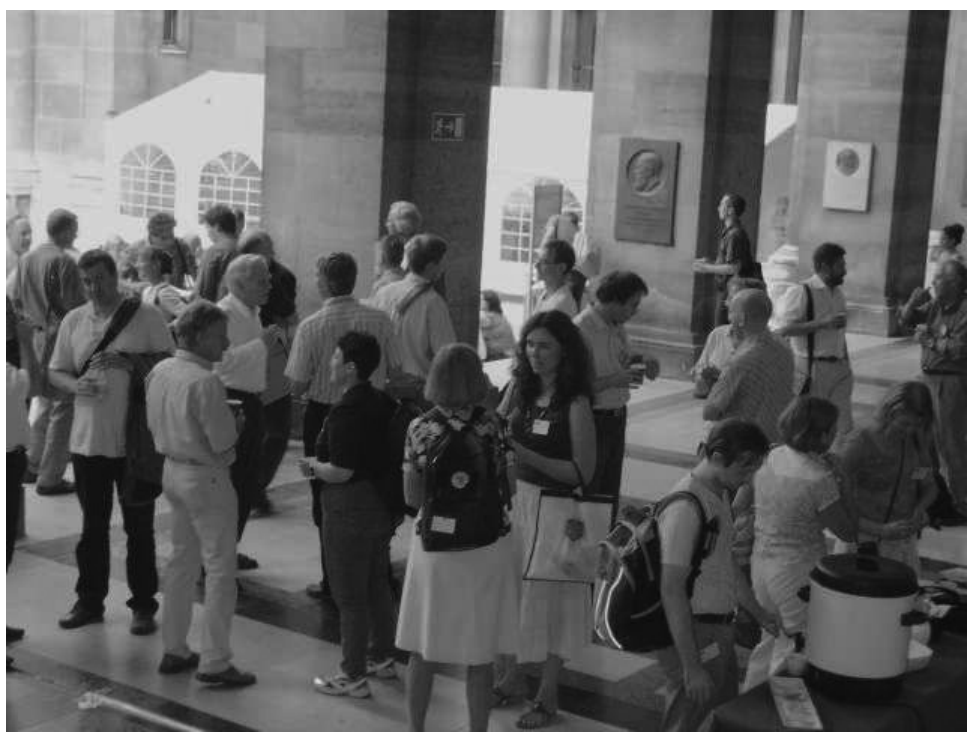



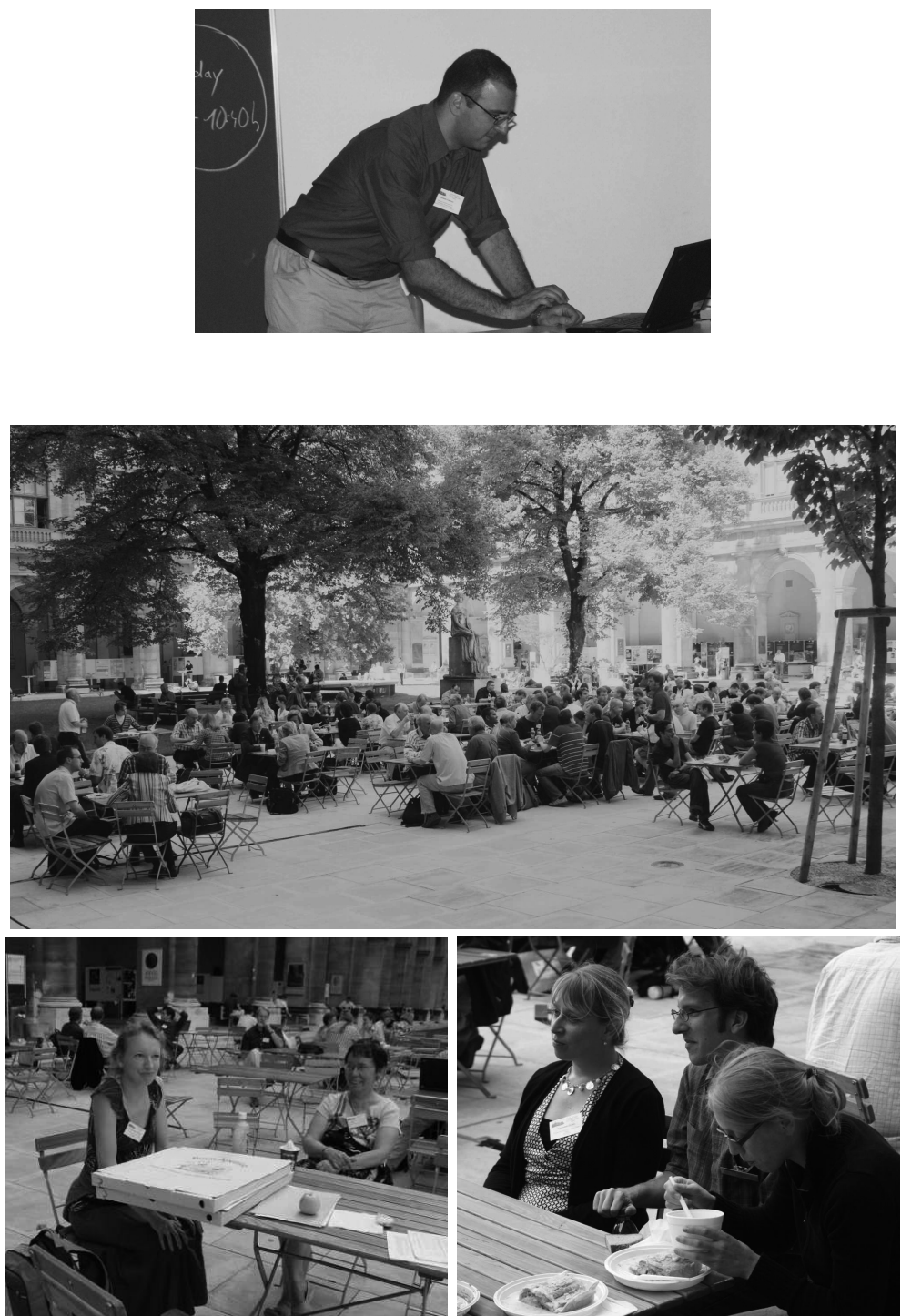

Lunch break in the Arcades of the main building of the university. 